\title{
Introduction to nonlinearities, business cycles, and forecasting
}

The first International Institute of Forecasters workshop on "Nonlinearities, business cycles and forecasting" took place on the 12th and 13th of December 2003 in Madrid. Nine invited papers by outstanding speakers were presented, followed by formal comments of invited discussants. In addition, there were lively discussions from the audience. This special issue provides reviewed versions of eight of these papers with additional comments by the discussants. Unfortunately the lecture by Mark Watson, entitled "Forecasting using empirical Bayes methods and a large number of predictors", was not made available for this special issue.

The overall theme of the workshop was motivated by a recently renewed interest in business cycles; see, e.g., the 2001 special issue of this Journal edited by Holden, Klein and Lahiri. Indeed, after several years where business cycles seemed outdated, the issue is recovering its flavour and the interest of academics, financial analysts, and even politicians. Logically, the complexities associated with businesscycle analysis have provoked a renewed interest in the measurement, implications and analysis of the cycle. Therefore the workshop tried to cover three fundamental issues:

1. How the cyclical information is extracted;

2. How this information should be analysed; and

3. What are the difficulties when using the cyclical indicators for monitoring and predicting the economic activity?

These questions are linked to a set of technical aspects such as the presence of nonlinearities (and the associated test statistics), the choice of the appropriate transformations to capture the cycles' asymmetries, the advantage in forecasting of modelling the aggregate versus modelling the individual series, dimensionality reduction procedures, etc.

On the other hand, understanding the sources of international economic fluctuations is important both for developing business-cycle models and for making policy. In this case, the issues of sample size and using cross-country panels become very relevant. For instance, if most of the variation in economic activity in a set of countries with different economic policies, institutions and economic structures is explained by a world business cycle, this lends support to the predictions of theoretical models emphasizing the common characteristics in the operations of markets rather than the differences in economic policies or institutional environments in those countries. Consequently, if a significant fraction of domestic business cycles is due to the common world factor, this implies that policies targeting external balances to stabilize sudden movements in economic activity might be ineffective. Is it true, as some recent and controversial findings claim, that the distinct "European" business cycle appears to be an artefact of limited samples? (Kose, Otrok, \& Whiteman, 2003). The interdisciplinary nature of the topics requires a combination of macroeconomic, statistical and econometric expertise. Against the above background we grouped the papers presented at the workshop into two different sets. The first four papers focus on nonlinearity in relation to the business cycle while the last four papers deal with various nonlinear time series models, model selection techniques, and their application to the analysis of nonlinearties in macroeconomic time series. 
The Zellner/Israilevich paper describes the origins and early development of the structural econometric modelling and time series analysis (SEMTSA) methodology/approach and its connection with the multisector Marshallian Macroeconomic Model (MMM). The authors review the past forecasting performance of the multivariate model. Then, using the SEMTSA approach, a discrete time one-sector MMM is presented with monetary and government sectors. Next the MMM is extended to n-sectors. A series of dynamic simulation experiments with various (nonlinear) versions of the MMM indicate that disaggregated data produces improved forecasts of aggregate, real GDP growth rates as well as sector forecasts. The discussant, Antoni Espasa, summarizes the main features of the SEMTSA/MMM approach. He points out the importance of incorporating seasonal effects and cointegration in the multi-sector MMM. Also, he stresses the need to detect and model the nonlinearities of aggregate variables by modeling those components which are clearly nonlinear, rather than by fitting a nonlinear model directly.

The Engel/Haugh/Pagan paper subjects the view that nonlinear models are important to an explanation of the business cycle to some critical analysis. They discuss ways of measuring the characteristics of the business cycle. A linear AR model and two univariate nonlinear models are fitted to US GDP. It appears that the two nonlinear models add little to the explanation for the asymmetry of phases. The discussant, Gabriel Pérez-Quirós, summarizes the main features of the paper and provides some general suggestions for further research.

In the Carvalho/Harvey paper a multivariate unobserved components (structural) model is fitted to eight US regional time series. A key feature of the proposed model is that it embodies convergence components which are able to display temporary divergence before converging to a common trend, also when the model is used for forecasting. Other theoretical issues of the $\mathrm{C} / \mathrm{H}$ paper are summarized by the discussants Jerez/Casals/Sotoca. They comment on the profile of the convergence components. In response to this comment and the final remark by Augustin Maravall, a new sub-section (2.2) on convergence was added to the paper. It makes the motivation behind the multivariate convergence model much clearer. In particular, the paragraph below Eq. (7) explains why convergence to a stationary process can be quite slow for the second-order error correction model; see also the forecasts of the US regions in Fig. 9 of the $\mathrm{C} / \mathrm{H}$ paper.

Kaiser/Maravall suggest that, in the extraction of smooth trends and cycles, the so-called ARIMAmodel-based (AMB) signal extraction procedure with the Hodrick-Prescott (HP) filter may solve the problems associated with the single application of both methods separately. The comments of the discussant, Javier Fernández-Macho, focus on two issues: (a) the HP filter can hardly be a solution to the drawbacks of the AMB approach, and (b) adding the ad-hoc HP filter to the AMB approach will yield "spurious and misleading" results. As an alternative the discussant suggests that the structural time series approach may provide a better solution in the sense that it is based on proper time series models for each component, thus allowing the use of classical procedures for testing. Both comments (a) and (b) are addressed by $\mathrm{K} / \mathrm{M}$ in detail in Section 5 of their paper.

Harvill/Ray present multi-step ahead forecasting results using univariate and multivariate functional coefficient AR (FCAR/VFCAR) models. FCAR models provide a fairly general, yet relatively simple, class of nonlinear time series models for practical use. There are several methods in the literature dealing with forecasting of general nonlinear time series processes. The authors restrict their comparison to three methods: naïve plug-in predictor, the bootstrap predictor, and the multi-stage predictor. Both simulation and empirical results indicate that the bootstrap method appears to give slightly more accurate forecast results for the data and models under study. The discussant, Nuno Crato, emphasizes the need for correctly specified nonlinear models and the effect of misperceived models on forecast evaluations. This requires further study and comparison of methods for identifying the functional structure of (V)FCAR and other nonlinear time series processes.

This latter issue is explored in more detail in the paper by Peña/Rodriguez. In particular, the authors explore the performance of various model selection criteria to obtain the order of the best fitted $\operatorname{AR}(p)$ model to the squared residuals of the linear model. Using four different linear model selection criteria (called "checks" by the discussants), they conclude that there is no evidence of nonlinearity if $p=0$. On 
the other hand, if $p>0$ there is a strong indication of nonlinearity. This is further checked by performing various linearity tests. Monte Carlo simulations suggest that BIC is a reasonable tool for detecting nonlinearity in time series. The discussants, Charles Bos and Ana Justel, summarize the main findings of the paper in their Table 2. They comment on the simulation set-up, and the iterative detection procedure. Instead of performing multiple linearity tests jointly, they propose a sequential testing procedure and illustrate its use through a small-scale simulation study.

The Teräsvirta/van Dijk/Medeiros paper is a very thorough evaluation study of the forecast accuracy of the (logistic) smooth transition autoregression model and two neural networks models using 47 monthly macroeconomic time series. Linear AR models are used as benchmarks. The number of topics discussed by the authors is quite extensive. Alfonso Novales, as discussant, summarizes seven topics in his introductory paragraph. He makes the important distinction between quantitative and qualitative forecast evaluation. If, as in the $\mathrm{T} / \mathrm{vD} / \mathrm{M}$ paper, the quantitative approach is followed, and a $5 \%$ (alternatively $2.5 \%$ ) difference in RMSEs indicates that one single model is better than another, no clear "winner" can be found. As a consequence, Novales stresses the need for a formal statistical test leading to a threshold for RMSE differences, as a function of statistical characteristics of the variable under study, different from the well-known Diebold-Mariano approach. Other interesting comments and suggestions for further research include the relationship between relative forecasting performance and forecast horizon, and the gains of forecast combinations from nonlinear models.

Can forecasts for macroeconomic aggregates like total output or total unemployment be improved by using a multi-level panel smooth transition AR (STAR) model for the disaggregated series? This is the key issue examined in the Fok/van Dijk/Franses paper. Based on simulation experiments and on comparisons of total US output forecasts with forecasts for US state-level output, they claim that improvements in one-step ahead forecasts can indeed be achieved. The discussant, Juan del Hoyo, points out some difficulties with the modelling process of STAR models. Given the complexity of the proposed panel STAR model, he suggests more research on the performance of the (time-consuming) ML estimation procedure. Since the differences in average MSPEs are minimal, the discussant also stresses the use of alternative forecasting evaluation methods like interval and density forecasts (one- and multi-step ahead).

\section{Acknowledgement}

We gratefully acknowledge financial support from the International Institute of Forecasters, Elsevier Science, The March Foundation, the Spanish Ministry of Science and Technology (SEC2002-10511-E), and the Autonóma de Madrid and Carlos III universities. We thank the editor of Macroeconomic Dynamics and Elsevier for allowing us to reproduce the Zellner/ Israilevich paper.

\section{Reference}

Kose, M. A., Otrok, C., \& Whiteman, C. H. (2003). International business cycles: World, region, and country specific factors. American Economic Review, 93(4), 12161239.

Antonio García-Ferrer

Departamento de Analisis Economico, Universidad Autonoma de Madrid, Cantoblanco, 28049 Madrid, Spain

Email address: Antonio.garcia@uam.es.

Jan G. De Gooijer Department of Quantitative Economics, University of Amsterdam, Roetersstraat 11, 1018 WB Amsterdam, The Netherlands Email address: j.g.degooijer@uva.nl. Corresponding author. Pilar Poncela

Departamento de Analisis Economico, Universidad Autonoma de Madrid, Cantoblanco, 28049 Madrid, Spain Email address: pilar.poncela@uam.es.

Esther Ruiz Department of Statistics and Econometrics, Universidad Carlos III de Madrid, Spain Email address: ortega@est-econ.uc3m.es. 